\title{
MicroRNA-34a expression levels in serum and intratumoral tissue can predict bone metastasis in patients with hepatocellular carcinoma
}

This article has been corrected. Correction in: Oncotarget. 2019; 10:6398-6399.

\author{
Zuo-Lin Xiang ${ }^{1, *}$, Xiao-Mei Zhao ${ }^{1, *}$, Li Zhang ${ }^{1}$, Ping Yang ${ }^{1}$, Jia Fan ${ }^{2}$, Zhao-You Tang ${ }^{2}$, \\ Zhao-Chong Zeng ${ }^{1}$ \\ ${ }^{1}$ Department of Radiation Oncology, Zhongshan Hospital, Fudan University, Shanghai, China \\ ${ }^{2}$ Department of Liver Cancer Institute, Zhongshan Hospital, Fudan University, Shanghai, China \\ * These authors have contributed equally to this work
}

Correspondence to: Zhao-Chong Zeng, email: zeng.zhaochong@zs-hospital.sh.cn

Keywords: hepatocellular carcinoma, bone metastasis, microRNA-34a, serum, tissue microarray

Received: July 05, 2016

Accepted: November 07, 2016

Published: November 23, 2016

\section{ABSTRACT}

Hepatocellular carcinoma (HCC) patients with bone metastasis (BM) suffer from pain and other symptoms that significantly reduce their quality of life. We screened a microRNA (miRNA) microarray to identify potential serum biomarkers for BM in HCC patients. A miRNA microarray was used to screen for BM-related miRNAs in paired serum samples from HCC patients with BM and from HCC patients without BM. Realtime quantitative polymerase chain reaction ( $($ RT-PCR) was used to quantify candidate miRNAs in serum samples from 106 independent HCC patients. Levels of candidate miRNAs in tissue samples from an independent cohort of 296 HCC patients were evaluated by in situ hybridization and intratumoral tissue microarray. The migration and invasion capabilities of HCCLM3 and SMMC-7721 cells were evaluated following treatment with a mimic and an inhibitor of miR-34a. Ninety miRNAs were differentially expressed in sera from HCC patients with BM when compared with sera from non-BM HCC patients $(P<0.05)$. Only miR-34a and miR-498 had false discovery rates (FDRs) $<0.05$. In cohorts of 106 and 296 HCC patients, we found that reduced serum and intratumoral miR-34a expression levels were independent risk factors for developing BM. Migration and invasion experiments indicated that a reverse correlation existed between miR-34a and HCC tumor migration and invasion. This study demonstrates the potential for the use of $\mathrm{miR}-34 \mathrm{a}$ as a serum and intratumoral tissue biomarker for predicting the risk of BM in HCC patients.

\section{INTRODUCTION}

Hepatocellular carcinoma (HCC) has an extremely poor prognosis and is the fifth most common cancer in men and the ninth in women worldwide. It is also the second leading cause of cancer death in men and the sixth in women worldwide [1]. More than $80 \%$ of HCC cases occur in sub-Saharan Africa and in Eastern Asia [2], and most are associated with liver cirrhosis as a result of chronic hepatitis B virus (HBV) or hepatitis $\mathrm{C}$ virus (HCV) infection. The frequency of bone metastasis (BM) in HCC patients with extrahepatic metastases was estimated to be $38.5 \%$ [3], whereas the frequency of BM in HCC patients that underwent curative resections was $11.7 \%$ [4]. During early metastasis, bone is destroyed by osteoclasts, which can cause pathological fractures, severe pain, and other nerve compression syndromes [5]. Bisphosphonates can inhibit the activity of osteoclasts and are used to reduce the frequency and severity of skeletal complications in BM patients [6]. Early prediction or diagnosis of BM in HCC patients is important for designing appropriate therapeutic regimens and assessing prognoses. If we can identify biomarkers to predict BM in HCC patients, we could take measures to reduce the probability that BM will develop and potentially enhance the quality of life for HCC patients.

We have determined that intratumoral connective tissue growth factor (CTGF), interleukin-11 (IL-11), and CXCR4 overexpression in primary tumor tissues is associated with BM in HCC patients $[4,7,8]$. We have 
also established a clinicopathologic model to predict $\mathrm{BM}$ in HCC patients [9], but this model is based on intratumoral tissue, which is inaccessible in many $\mathrm{HCC}$ patients. On the other hand, a serum biomarker screen combined with a predictive model could be used for every HCC patient. Further, microRNA (miRNA) is more stable than mRNA in formalin-fixed, paraffin-embedded (FFPE) specimens and is easily obtained from patient serum. Thus, we investigated whether serum and intratumoral tissue miRNAs could be used to predict BM in HCC patients.

\section{RESULTS}

\section{Serum miRNA microarray analysis}

We identified 90 miRNAs that were differentially expressed in the BM and non-BM groups (Supplementary Table S1). In serum samples from the BM group, 27 miRNAs were upregulated and 63 miRNAs were downregulated when compared with serum samples from the non-BM group (Figure 1). Among the 90 miRNAs, only two had FDR values $<0.05$; miR-34a was downregulated and had an FDR of 0.020 and miR-498 was upregulated and had an FDR of 0.018 . MiR-34a and miR498 were further analyzed in an independent cohort of 106 HCC patients to determine whether they could serve as potential serum biomarkers to predict BM.

\section{qPCR confirmed serum miR-34a as a risk factor for BM}

To further evaluate the potential of miR-34a and miR-498 to serve as biomarkers, qPCR was performed to quantify their levels in the sera of $106 \mathrm{HCC}$ patients. The results of the univariate analyses revealed that serum miR-34a $(P=0.002)$, serum miR-498 $(P=0.038)$, tumor differentiation $(P=0.011)$, tumor number $(P=0.035)$, vascular invasion $(P=0.005)$, and BCLC stage $(P=0.002)$ were risk factors for $\mathrm{BM}$ in $\mathrm{HCC}$ patients (Table 1). The results of the multivariate analyses revealed that serum

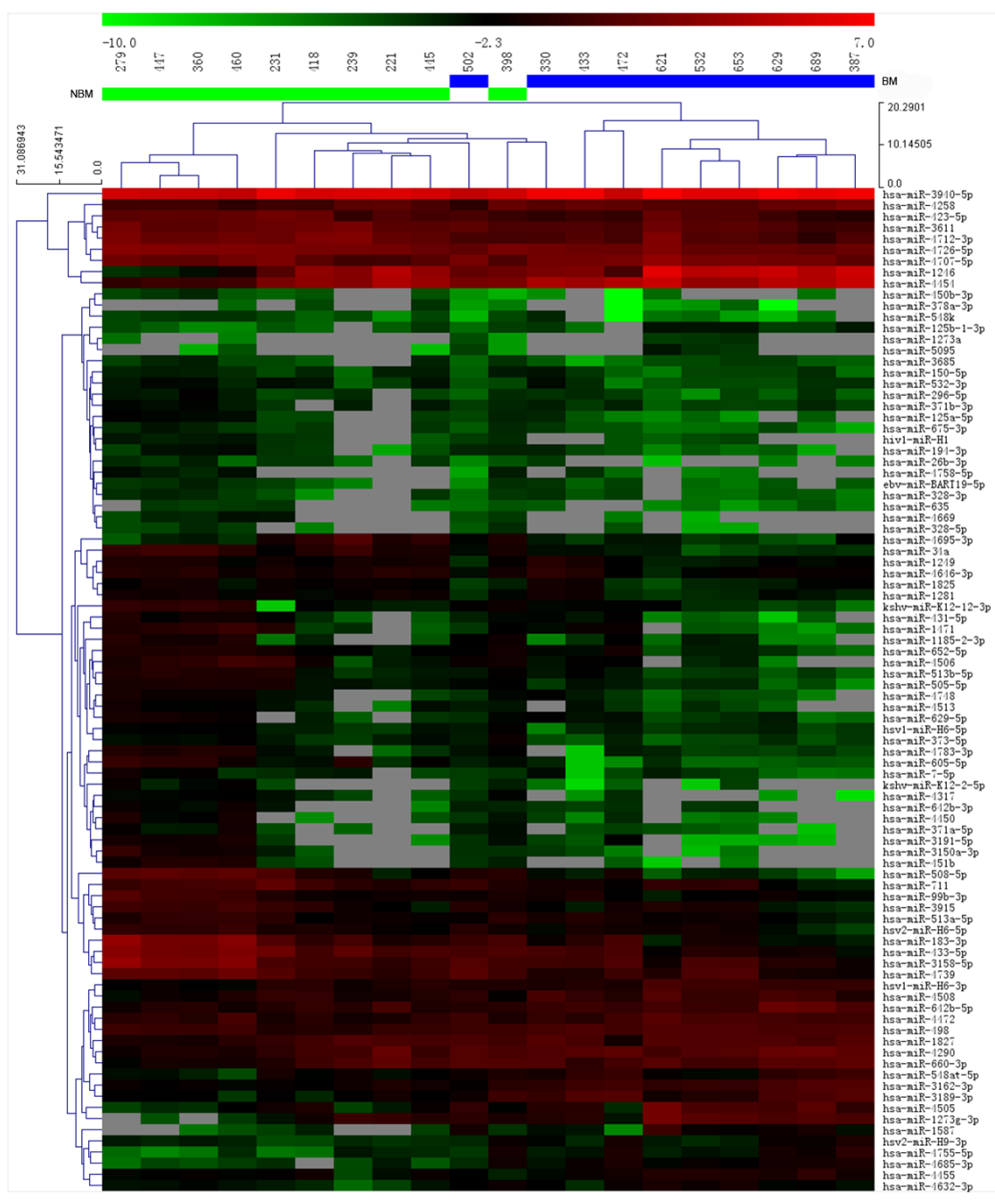

Figure 1: Hierarchical clustering of 90 differentially expressed miRNAs. Hierarchical clustering of miRNAs that were differentially expressed in the sera of HCC patients with BM vs. non-BM HCC patients. Red indicates high relative expression and green indicates low relative expression. 
Table 1: Univariate analyses of factors associated with bone metastasis in a cohort of $106 \mathrm{HCC}$ patients

\begin{tabular}{|c|c|c|}
\hline \multirow{2}{*}{ Variable } & \multicolumn{2}{|c|}{ Bone metastasis } \\
\hline & HR $(95 \%$ CI $)$ & $P$ \\
\hline Age $(\leq 51$ versus $>51$ years $)$ & $2.488(0.779-7.943)$ & 0.124 \\
\hline Gender (male versus female) & $0.834(0.186-3.739)$ & 0.813 \\
\hline HBsAg (negative versus positive) & $3.824(0.499-29.277)$ & 0.197 \\
\hline $\mathrm{HCV}-\mathrm{Ab}$ (negative versus positive) & $3.636(0.467-28.289)$ & 0.218 \\
\hline AFP, $n g / m L(\leq 20$ versus $>20)$ & $0.628(0.210-1.876)$ & 0.404 \\
\hline ALT, U/L $(\leq 40$ versus $>40)$ & $1.188(0.412-3.427)$ & 0.750 \\
\hline$\gamma-\mathrm{GT}, \mathrm{U} / \mathrm{L}(\leq 50$ versus $>50)$ & $1.549(0.486-4.942)$ & 0.460 \\
\hline Liver cirrhosis (no versus yes) & $2.631(0.343-20.171)$ & 0.352 \\
\hline Child-Pugh score (A versus B) & $0.048(0.000-7.243 \mathrm{E} 6)$ & 0.752 \\
\hline Tumor differentiation (I-II versus III-IV) & $3.954(1.323-11.810)$ & 0.011 \\
\hline Tumor size, $\mathrm{cm}(\leq 5$ versus $>5)$ & $2.186(0.730-6.545)$ & 0.162 \\
\hline Tumor number (single versus multiple) & $3.120(1.081-8.999)$ & 0.035 \\
\hline Tumor encapsulation (none versus complete) & $1.227(0.430-3.502)$ & 0.702 \\
\hline Vascular invasion (no versus yes) & $4.611(1.599-13.296)$ & 0.005 \\
\hline BCLC stage (0-A versus B-C) & $5.599(1.916-16.363)$ & 0.002 \\
\hline Serum miR-34a (high versus low) & $26.722(3.478-205.288)$ & 0.002 \\
\hline Serum miR-498 (low versus high) & $3.870(1.078-13.887)$ & 0.038 \\
\hline
\end{tabular}

NOTE: Univariate analysis and Cox proportional hazards regression model. Tumor differentiation evaluated was based on the Edmondson-Steiner grading.

Abbreviation: $\mathrm{HBsAg}$, hepatitis B virus surface antigen; $\mathrm{HCV}-\mathrm{Ab}$, hepatitis $\mathrm{C}$ virus antibody; AFP, alpha-fetoprotein; ALT, alanine aminotransferase; $\gamma$-GT, $\gamma$-glutamyl-transferase; BCLC, Barcelona Clinic Liver Cancer

miR-34a $(P=0.010)$, vascular invasion $(P=0.047)$, and BCLC stage $(P=0.039)$ were independent risk factors for BM in HCC patients (Table 2). Of the two miRNAs, only miR-34a predicted BM in HCC patients. At follow-up, 14 of the 106 HCC patients developed BM. Among the 14 BM patients, 13 patients exhibited down-regulated miR34a levels.

\section{Correlations between miR-34a expression in intratumoral tissue and clinicopathologic features}

ISH and intratumoral TMAs from $296 \mathrm{HCC}$ patients were used to determine whether intratumoral miR34a could predict BM in HCC patients. MiR-34a was primarily localized to the cytoplasm and nuclei of tumor cells (Supplementary Figure S1). Intratumoral miR-34a was expressed in $219(74.0 \%)$ patients. The associations between miR-34a expression and clinicopathologic factors were analyzed (Supplementary Table S2). At follow-up, 91 patients developed extrahepatic metastases. Of these 91 patients, 37 developed BM, 46 developed lung metastasis,
39 developed lymph node metastasis, 9 developed adrenal gland metastasis, and 3 developed brain metastasis. Patients with multiple organ metastases were included in every applicable organ group. We analyzed whether or not there was a correlation between the expression of miR-34a and different organ metastasis and determined that intratumoral miR-34a expression was negatively correlated with BM $(r=-0.405 ; P<0.001)$, but was not correlated with lung metastasis $(r=-0.094 ; P=0.105)$, lymph node metastasis $(r=-0.087 ; P=0.137)$, adrenal gland metastasis $(r=-0.130 ; P=0.824)$, or brain metastasis $(r=-0.380 ; P=0.512)$. Intratumoral miR-34a expression was also correlated with BCLC stage $(r=-0.115 ; P=$ $0.048)$ and vascular invasion $(r=-0.121 ; P=0.038)$, but did not correlate with other clinicopathologic factors (age, gender, HBsAg, HCV-Ab, AFP, ALT, $\gamma$-GT, liver cirrhosis, Child-Pugh score, tumor differentiation, tumor size, tumor number, or tumor encapsulation). We also found that BCLC stage $(r=0.194 ; P=0.001)$, vascular invasion $(r=$ $0.256 ; P<0.001)$, tumor number $(r=0.118 ; P=0.042)$, and tumor differentiation $(r=0.163 ; P<0.005)$ were positively correlated with BM. 
Table 2: Multivariate analyses of factors associated with bone metastasis in a 106 hepatocellular carcinoma patient cohort

\begin{tabular}{lcc}
\hline \multirow{2}{*}{ Variable } & \multicolumn{2}{c}{ Bone metastasis } \\
\cline { 2 - 3 } Tumor differentiation (I-II versus III-IV) & $1.751(0.552-5.556)$ & 0.342 \\
Tumor number (single versus multiple) & $1.728(0.540-5.528)$ & 0.356 \\
Vascular invasion (no versus yes) & $3.078(1.013-9.351)$ & 0.047 \\
BCLC stage (0-A versus B-C) & $3.471(1.068-11.286)$ & 0.039 \\
Serum miR-34a (high versus low) & $16.034(1.915-134.265)$ & 0.010 \\
Serum miR-498 (low versus high) & $1.978(0.442-8.839)$ & 0.372
\end{tabular}

NOTE: Variables were adopted for their prognostic significance on univariate analysis. Tumor differentiation evaluated was based on the Edmondson-Steiner grading.

Abbreviation: BCLC, Barcelona Clinic Liver Cancer.

\section{Correlations between intratumoral miR-34a expression and BM, non-BM, and overall metastasis}

At the last follow-up for the group of $296 \mathrm{HCC}$ patients, $91(30.7 \%)$ of the patients presented with extrahepatic metastases including 37 (12.5\%) with BM. Among the $37 \mathrm{BM}$ patients, 31 patients exhibited downregulated miR-34a levels. At five years, the BM-free survival rate was $49.9 \%$, the progression-free survival rate was $45.9 \%$, and the overall survival rate was $50.5 \%$. The results of the log-rank test indicated that a lower intratumoral miR-34a expression level was more highly associated with increased incidence of BM than a higher miR-34a expression level $(P<0.001)$. The median BM free survival time determined by the Kaplan-Meier and logrank tests in patient groups with high miR-34a expression was 39.9 months, whereas the corresponding survival time in patients with low miR-34a expression was 17.8 months $(P<0.001)$.

Cox regression univariate analyses revealed that tumor differentiation $(P=0.011)$, tumor number $(P=0.032)$, vascular invasion $(P<0.001)$, BCLC stage $(P=0.001)$, and the intratumoral miR-34a level $(P<0.001)$ were associated with BM (Table 3). Cox regression multivariate analyses identified vascular invasion $(P=0.003)$, BCLC stage $(P=0.011)$, and the intratumoral miR-34a level $(P<0.001)$ as independent risk factors for BM (Table 4).

For the 54 patients who developed metastases (lung, lymph node, adrenal gland, and brain metastases) that did not involve the bone (non-BM), Kaplan-Meier and logrank tests indicated that the level of intratumoral miR-34a expression did not affect the rate of metastases in nonBM patients $(P=0.521)$. The rate of overall metastases was higher in HCC patients with lower levels of miR-34a expression $(P<0.001)$.

\section{MiR-34a negatively regulates $\mathrm{HCC}$ cell proliferation, migration, and invasion}

Among the four HCC cell lines (SMMC-7721, HepG2, MHCC97H, and HCCLM3) with an ascending order of metastatic potentials (Supplementary Figure S2A), we chose SMMC-7721 cells, which exhibited the lowest degree of invasion and HCCLM3 cells, which exhibited the highest degree of invasion, for the migration and invasion assay. Further, their endogenous level of miR-34a was reversely attenuated (Supplementary Figure S2B), which indicated miR-34a may affect HCC metastasis.

MiR-34a expression in cells was induced or inhibited by transfection with a miR-34a mimic or a miR$34 \mathrm{a}$ inhibitor, respectively. As well, the results of the qPCR analysis demonstrated that transfection with $100 \mathrm{nM}$ of the miR-34a inhibitor decreased miR-34a expression 5.0-fold in the SMMC-7721 cells (Supplementary Figure S2C), whereas transfection with $100 \mathrm{nM}$ of the miR-34a mimic increased miR-34a expression 5.7-fold in the HCCLM3 cells (Supplementary Figure S2D).

To study the role miR-34a played in $\mathrm{HCC}$, we first investigated the effect of miR-34a on HCC proliferation using the Cell Counting Kit- 8 assay, the results of which indicated that compared with the negative controls, the ability of cells to proliferate was slightly accelerated when the SMMC-7721 cells were transfected with the miR-34a inhibitor, whereas the proliferation ability was significantly inhibited when the HCCLM3 cells were transfected with the miR-34a mimic (Figure 2A, 2B). These findings indicated that miR-34a negatively regulated $\mathrm{HCC}$ cell proliferation.

In the SMMC7721 and HCCLM3 cell migration assays, the migration capabilities of the SMMC-7721 cells were increased 3.51-fold after transfection with 
Table 3: Univariate analyses of factors associated with bone metastasis in a cohort of 296 HCC patients

\begin{tabular}{|c|c|c|}
\hline \multirow{2}{*}{ Variable } & \multicolumn{2}{|c|}{ Bone metastasis } \\
\hline & HR $(95 \%$ CI $)$ & $P$ \\
\hline Age $(\leq 51$ versus $>51$ years $)$ & $0.867(0.454-1.655)$ & 0.665 \\
\hline Gender (male versus female) & $0.808(0.286-2.280)$ & 0.687 \\
\hline HBsAg (negative versus positive) & $1.244(0.547-2.834)$ & 0.602 \\
\hline $\mathrm{HCV}-\mathrm{Ab}$ (negative versus positive) & $3.455(0.333-18.094)$ & 0.378 \\
\hline AFP, $n g / m L(\leq 20$ versus $>20)$ & $1.061(0.524-2.148)$ & 0.869 \\
\hline ALT, U/L $(\leq 40$ versus $>40)$ & $0.885(0.455-1.720)$ & 0.719 \\
\hline$\gamma-\mathrm{GT}, \mathrm{U} / \mathrm{L}(\leq 50$ versus $>50)$ & $1.518(0.760-3.034)$ & 0.237 \\
\hline Liver cirrhosis (no versus yes) & $2.138(0.656-6.965)$ & 0.207 \\
\hline Child-Pugh score (A versus B) & $0.049(0.000-5.708 \mathrm{E} 5)$ & 0.717 \\
\hline Tumor differentiation (I-II versus III-IV) & $2.304(1.209-4.391)$ & 0.011 \\
\hline Tumor size, $\mathrm{cm}(\leq 5$ versus $>5)$ & $1.546(0.806-2.965)$ & 0.190 \\
\hline Tumor number (single versus multiple) & $2.041(1.064-3.915)$ & 0.032 \\
\hline Tumor encapsulation (none versus complete) & $0.690(0.358-1.331)$ & 0.268 \\
\hline Vascular invasion (no versus yes) & $4.516(2.362-8.632)$ & $<0.001$ \\
\hline BCLC stage (0-A versus B-C) & $3.198(1.627-6.285)$ & 0.001 \\
\hline Intratumoral miR-34a (positive versus negative) & $8.005(3.874-16.541)$ & $<0.001$ \\
\hline
\end{tabular}

NOTE: Univariate analysis and Cox proportional hazards regression model. Tumor differentiation evaluated was based on the Edmondson-Steiner grading.

Abbreviation: HBsAg, hepatitis B virus surface antigen; HCV-Ab, hepatitis C virus antibody; AFP, alpha-fetoprotein; ALT, alanine aminotransferase; $\gamma$-GT, $\gamma$-glutamyl-transferase; BCLC, Barcelona Clinic Liver Cancer.

Table 4: Multivariate analyses of factors associated with bone metastasis in a 296 hepatocellular carcinoma patient cohort

\begin{tabular}{lcc}
\hline \multirow{2}{*}{ Variable } & \multicolumn{2}{c}{ Bone metastasis } \\
\cline { 2 - 3 } & HR (95\% CI) & P \\
\hline Tumor differentiation (I-II versus III-IV) & $1.613(0.822-3.163)$ & 0.164 \\
Tumor number (single versus multiple) & $1.707(0.848-3.434)$ & 0.134 \\
Vascular invasion (no versus yes) & $2.773(1.427-5.387)$ & 0.003 \\
BCLC stage (0-A versus B-C) & $2.464(1.226-4.949)$ & 0.011 \\
Intratumoral miR-34a(positive versus negative) & $6.011(2.857-12.645)$ & $<0.001$ \\
\hline
\end{tabular}

NOTE: Variables were adopted for their prognostic significance on univariate analysis. Abbreviation: BCLC, Barcelona Clinic Liver Cancer.

the miR-34a inhibitor compared with negative control oligonucleotide-transfected SMMC-7721 cells $(P=0.0004$; Figure 2C, 2D). After transfection with the miR-34a mimic, the migration capabilities of the HCCLM3 cells were reduced 2.58-fold compared with the migration of the negative control oligonucleotide-transfected HCCLM3 cells ( $P=0.0012$; Figure 2C, 2E).
In invasion assays with SMMC7721 and HCCLM3 cells, the invasion capabilities of the SMMC-7721 cells were increased 2.59-fold after transfection with the miR-34a inhibitor compared with negative control oligonucleotide-transfected SMMC-7721 cells $(P=0.0048$. Figure 2F, 2G). After transfection with the miR-34a mimic, the invasion capabilities of HCCLM3 cells were 
A

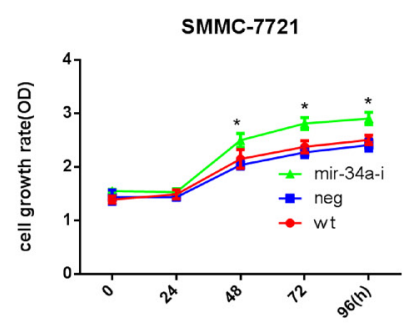

B

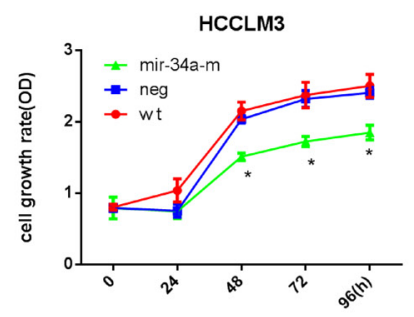

C

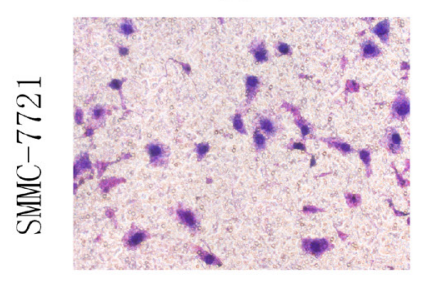

WT
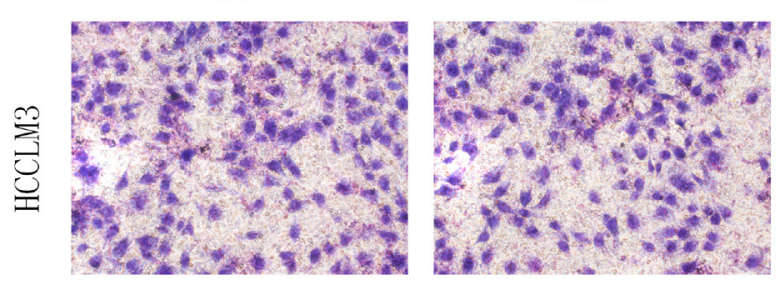

miR-34a-i

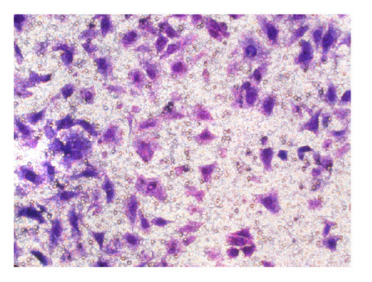

mi R-34a-m

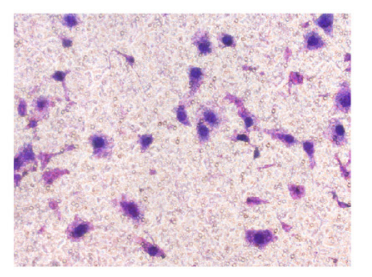

D

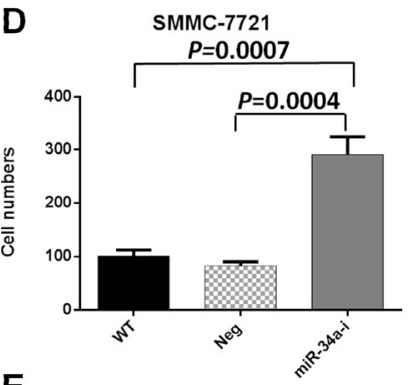

E
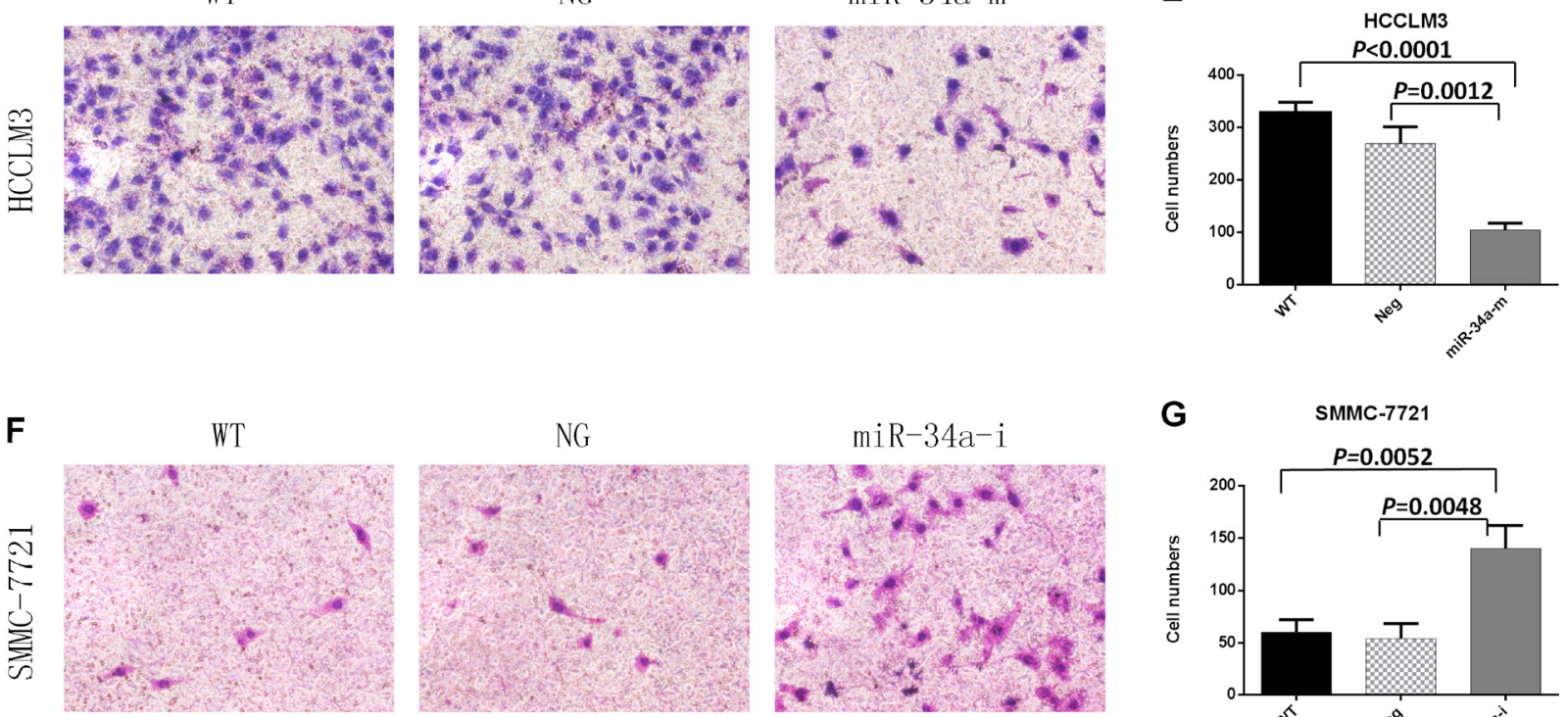

NG
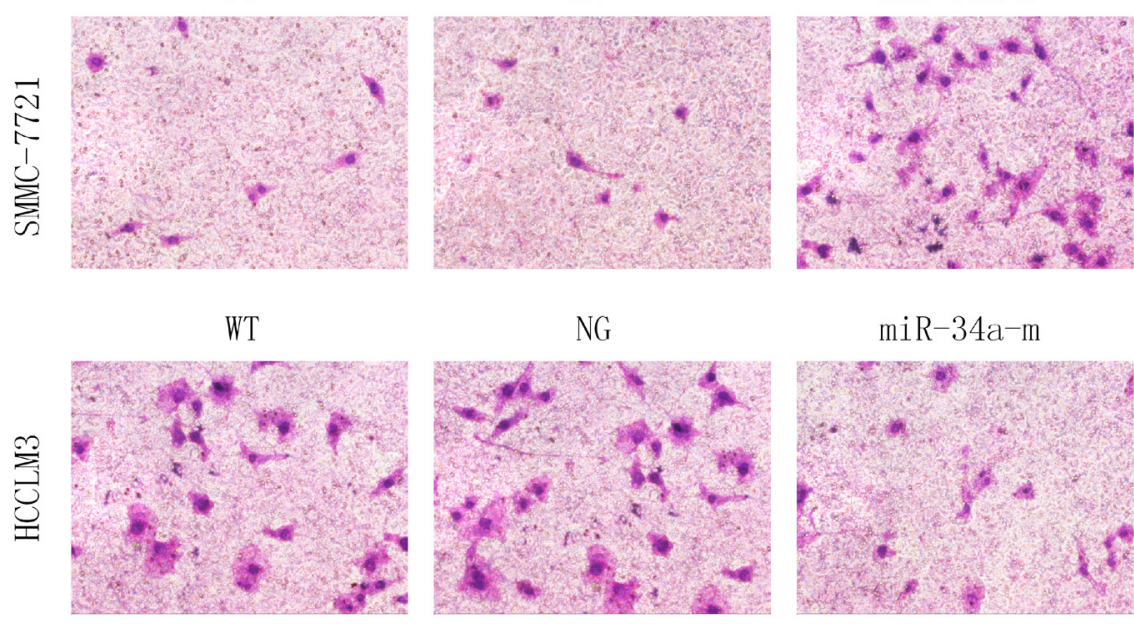

NG

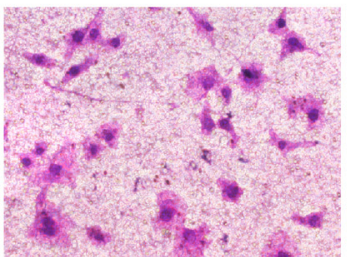

miR-34a-m

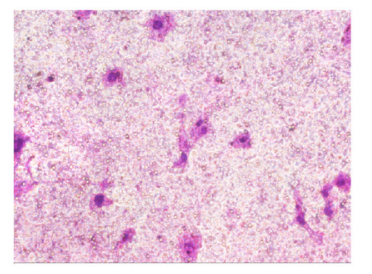

G

SMMC-7721

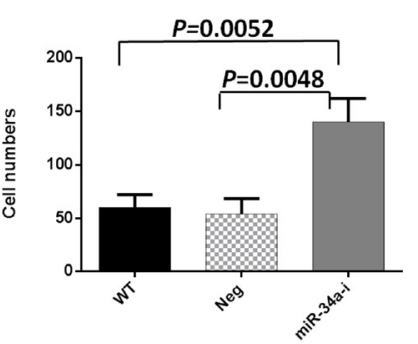

H

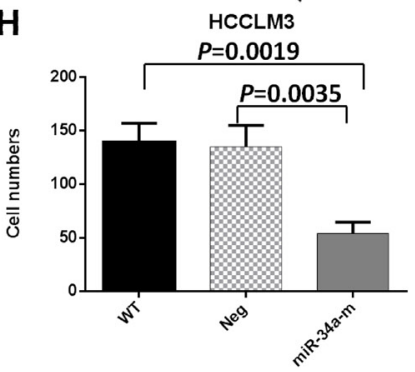

Figure 2: Proliferation, migration, and invasion assays of SMMC-7721 and HCCLM3 cells after transfection with the indicated oligonucleotides. WT, NG, miR-34a-m, and miR-34a-i indicate non-transfected, negative control oligonucleotidetransfected, miR-34a mimic- transfected, and miR-34a inhibitor-transfected cells, respectively. A. SMMC-7721 cell growth rates after transfection with the miR-34a inhibitor. B. HCCLM3 cell growth rates after transfection with the miR-34a mimic. $*=P<0.05$, compared to negative controls at the same time point. C. Representative images of the migration assay. D. and E. Numbers of migrating cells on the undersides of the membranes. Data are presented as mean \pm SEM and are representative of three independent experiments. The images were acquired at a magnification of $200 \times$. F. Representative images of the invasion assay. G. and $\mathbf{H}$. Numbers of invading cells on the undersides of the membranes. Data are presented as mean \pm SEM and are representative of three independent experiments. The images were acquired at a magnification of $200 \times$. 
reduced 2.50-fold compared with the invasion of the negative control oligonucleotide-transfected HCCLM3 cells $(P=0.0035$ Figure $2 \mathrm{~F}, 2 \mathrm{H})$.

\section{DISCUSSION}

We analyzed serum and intratumoral tissues from HCC patients to investigate differential miRNA expression in HCC patients with BM and without BM. We found 90 miRNAs that were differentially expressed in patients with BM compared with non-BM patients. Of the 90 miRNAs, only miR-34a and miR-498 had FDRs $<0.05$. We further determined that miR-34a levels in serum and intratumoral tissue predicted BM in HCC patients. BM is a common cause of pain and the source of other symptoms that reduce the quality of life for HCC patients [10]. A screening method to predict the risk of BM in HCC patients would allow for improved treatments for HCC patients with a high risk of BM. For example, patients with an increased risk of BM could be treated with bisphosphonates to prevent BM.

Overexpression of miR-34a reportedly suppresses tumor progression and leads to improved prognoses, whereas reduced miR-34a expression is associated with poor overall survival in several cancers [11-17]. The association between miR-34a levels and glioma has been more ambiguous. Gao et al. found that lower miR-34a expression was correlated with reduced progression-free survival and overall survival in glioma patients [18], but Genovese et al. found that lower miR-34a expression led to improved overall survival in glioblastoma patients [19]. In this study, we found that low levels of miR-34a in serum and intratumoral tissue were associated with increased incidence of BM in HCC patients. We also found that low levels of miR-34a in intratumoral tissue increased BCLC stage and vascular invasion in HCC patients.

Serum miRNAs have been used as noninvasive biomarkers for many cancers [20-23]. MiR-34 has also been found to suppress osteoclasts and has potential as a therapeutic to protect the skeleton and to ameliorate BM [24]. Further, Chen et al. found that miR-34a inhibited BM in Ras-activated prostate cancer [25], and Gougelet et al. found that miR-34a functioned as an inhibitor and had antitumor activity in liver cancer [26]. Yang et al. found a correlation between miR-34a levels and venous metastasis in HBV-positive HCC patients [27]. We found that low miR-34a levels in serum and intratumoral tissue were associated with increased incidence of BM in $\mathrm{HCC}$ patients and that miR-34a expression can be used to identify HCC patients at high risk for developing BM.

We believe that miR-34a blocks osteoporosis and $\mathrm{BM}$ by inhibiting osteoclastogenesis in HCC patients because miR-34a has been found to directly target transforming growth factor- $\beta$-induced factor 2 (TGIF2) and to reduce bone resorption [24]. Because a reduction in miR-34a may be linked to a high level of TGF- $\beta$ signaling activity [27], we speculate that miR-34a contributes to BM in HCC via a TGF- $\beta$-miR-34a signaling pathway. MiR34 a may be used as a biomarker to predict BM in HCC patients; thus, miR-34a could aid in clinical decisionmaking.

In conclusion, this study demonstrated that low miR-34a expression in serum and in intratumoral tissue are independent risk factors for developing BM in HCC patients. Our findings suggest that miR-34a may be important for facilitating BM in patients with HCC. Nonetheless, further study is required to elucidate the mechanism by which low miR-34a increases the development of BM in $\mathrm{HCC}$ patients.

\section{MATERIALS AND METHODS}

\section{Clinical studies}

Ten HCC patients underwent curative resection at Zhongshan Hospital and presented with BM, but had no additional metastases. Blood samples from these patients were collected at the time of surgery. The matched nonBM patients included 10 HCC patients who did not develop metastases 5 years after curative resection. Blood samples from these patients were collected at Zhongshan Hospital between February 2008 and January 2010. To obtain serum samples, whole blood samples were centrifuged at $1000 \times g$ for $10 \mathrm{~min}$ at $4^{\circ} \mathrm{C}$. Serum supernatants were then transferred to new tubes and stored at $-80^{\circ} \mathrm{C}$.

We used age, gender, hepatitis B surface antigen ( $\mathrm{HBsAg}$ ), hepatitis $\mathrm{C}$ antibody (HCV-Ab), $\alpha$-fetoprotein, alanine transaminase, $\gamma$-glutamyl-transferase, liver cirrhosis, Child-Pugh score, tumor differentiation, tumor size, tumor number, tumor encapsulation, vascular invasion, and Barcelona Clinic Liver Cancer (BCLC) stage data to match the BM group with a non-BM group. These factors did not differ significantly between the two groups. Clinicopathologic characteristics are summarized in Supplementary Table S3.

An additional study involving an independent cohort of $106 \mathrm{HCC}$ patients was conducted to evaluate the clinical significance of candidate miRNAs identified in our preliminary serum microarray study. All of the 106 patients underwent a hepatectomy at Zhongshan Hospital. Blood samples were collected from the patients between August 2008 and September 2011 before their hepatectomies. None of the patients had distant metastases at the time of blood collection. To exclude the possibility of extrahepatic spread, each patient underwent a chest $\mathrm{X}$-ray and abdominal ultrasonography before surgery, and a bone scan was performed if the patient reported bone pain. If extrahepatic spread was suspected, computed tomography and/or magnetic resonance imaging (MRI) were used to determine whether extrahepatic spread had occurred. Additional inclusion criteria were as follows: 
HCC diagnosis based on pathology, no prior anticancer treatment, acceptable blood sample, and complete clinicopathologic follow-up data. The tumor stage was determined according to the BCLC staging system. The histologic grade of tumor differentiation was assigned using the Edmondson grading system. Tumor size was determined by measuring the largest dimension of the tumor specimen. The extent of vascular invasion was determined by microscopic examination of the resected specimen. The detailed clinicopathologic features of these patients are provided in Supplementary Table S4.

The clinical significance of the candidate biomarker was evaluated using intratumoral tissues from an independent cohort of 296 consecutive HCC patients who did not have distant metastasis before surgery. All of these patients underwent a hepatectomy between August 2002 and September 2007 at the Liver Cancer Institute, Zhongshan Hospital, Fudan University. None of these patients received any preoperative anticancer treatment, and prior to surgery, each patient underwent a chest X-ray and abdominal ultrasonography to exclude extrahepatic metastases. The clinicopathological characteristics of these patients are summarized in Supplementary Table S5.

Study protocols were approved by the Zhongshan Hospital Research Ethics Committee. Informed consent was obtained from each patient in accordance with the committee regulations.

\section{Follow-up and postoperative treatment}

Serum samples were collected from $10 \mathrm{HCC}$ patients that presented with $\mathrm{BM}$ but had no additional metastases. The matched non-BM group included 10 HCC patients who also had no additional metastases at follow-up; sera from the non-BM patients were collected at follow-up. The median follow-up time was 65 months with a range of 60-73 months.

The independent cohort of $106 \mathrm{HCC}$ patients received their follow-ups by August 2015, and the median follow-up time was 41.9 months with a range of 4.4-84 months.

The clinical significance of the candidate miRNA was evaluated using intratumoral tissues from an independent cohort of 296 consecutive HCC patients. These patients were observed until December 2012. The median follow-up time for this group was 54.6 months with a range of 3.2-124.1 months. At each check-up, a medical history was recorded and a physical examination was performed. A chest radiograph was performed every 6 months, ultrasonography of the abdomen was performed every 3 months, and laboratory tests were performed every 3 months. Time to recurrence was calculated from the date of the operation to the date of recurrence. BM was diagnosed as previously described [4]. Briefly, a bone scan was performed annually. If a patient reported localized bone pain, a bone scan or an MRI was performed immediately. A BM diagnosis was based on a history of $\mathrm{HCC}$, the presence of symptoms, and radiological imaging studies. Time to BM was calculated from the date of the operation to the date of BM. When a diagnosis of BM was made, the affected bone was treated with external beam radiotherapy. Metastases at other locations were treated with radiotherapy, interventional therapy, or surgery.

\section{MiRNA microarray analysis}

Serum samples from 20 patients (10 BM and 10 non-BM) were used for miRNA profiling. Profiling was performed using the 7 th generation miRCURY ${ }^{\mathrm{TM}}$ LNA Array (v.18.0, Exiqon, Vedbaek, Denmark) and microarray experiments were performed by KangChen Bio-tech (Shanghai, China). Briefly, total RNA was harvested using TRIzol ${ }^{\circledR}$ LS reagent (Invitrogen) and a miRNeasy mini kit (Qiagen) according to the manufacturer's instructions. In detail, $0.75 \mathrm{ml}$ TRIzol ${ }^{\circledR} \mathrm{LS}$ reagent was added to $0.25 \mathrm{ml}$ serum samples. Chloroform $(0.2 \mathrm{ml})$ was added for phase separation, 1.5 volumes of $100 \%$ ethanol were added to the aqueous phase, and the mixture was loaded onto a miRNeasy column according to the manufacturer's instructions. RNA samples were quantified using a NanoDrop ${ }^{\circledR} 1000$ (ND-1000, NanoDrop Technologies), labeled using the miRCURY LNA ${ }^{\mathrm{TM}}$ Hy3 ${ }^{\mathrm{TM}} / \mathrm{Hy} 5^{\mathrm{TM}}$ Power labeling kit, and hybridized on the miRCURY LNA ${ }^{\mathrm{TM}}$ Array (v.18.0). After washing, the slides were scanned using the Axon GenePix ${ }^{\circledR}$ 4000B microarray scanner. Scanned images were imported into GenePix Pro 6.0 software (Axon) for grid alignment and data extraction. Replicated miRNAs were averaged and miRNAs with intensities $\geq 30$ in all samples were used to calculate the normalization factor according to the median normalization method. After normalization, significant differentially expressed miRNAs were identified by Volcano Plot filtering. Finally, hierarchical clustering was performed using MEV software (v.4.6, TIGR) to identify differences in miRNA expression profiles among samples.

\section{RNA isolation and $q R T-P C R$}

Total RNA was isolated using TRIzol ${ }^{\circledR}$ LS reagent (Invitrogen) and a miRNeasy mini kit (Qiagen) according to manufacturer's instructions. Briefly, 3 volumes $(0.75 \mathrm{ml})$ of TRIzol ${ }^{\circledR} \mathrm{LS}$ reagent was added to a serum sample $(0.25 \mathrm{ml}), 25 \mathrm{fmol}$ of cel-miR-39 was added for normalization [28], $0.2 \mathrm{ml}$ of chloroform was added, and each sample was centrifuged. After centrifugation, the aqueous phase was transferred to a new tube, 1.5 volumes of $100 \%$ ethanol were added, and the mixture was loaded onto a miRNeasy column (Qiagen) according to the manufacturer's instructions. The final elution volume ranged from 20-30 $\mu$ l. RNA sample concentrations were quantified using a NanoVue Plus spectrophotometer (GE, General Electric Company). Serum RNA (500 
ng, including miRNA) was used for first-strand DNA synthesis with an All-in-One ${ }^{\mathrm{TM}}$ First-Strand cDNA Synthesis kit (GeneCopoeia Inc.). Real-time PCR was performed in triplicate using the SYBR ${ }^{\circledR}$ Green PCR method and the All-in-One ${ }^{\mathrm{TM}}$ miRNA qPCR Detection kit (GeneCopoeia Inc.). The expression levels of serum miRNAs were normalized against cel-miR-39 using the $2^{-\Delta \Delta \mathrm{Ct}}$ method [29]. Total RNA was isolated from HCC cell lines using TRIzol ${ }^{\circledR}$ (Invitrogen) and miR-34a expression was normalized against U6 expression. Expression analysis was performed using a 7500 Real-Time PCR System (Applied Biosystems).

\section{Tissue microarray}

A tissue microarray (TMA) was constructed as previously described [30-32]. Hematoxylin and eosin (H\&E)-stained slides were screened to identify the optimal intratumoral tissue for analysis. The TMA slides were then constructed (in collaboration with the Shanghai Biochip Company Ltd.) using samples from the cohort of 296 HCC patients. From each FFPE HCC tissue sample, two punch cores (longest dimension $=1.0 \mathrm{~mm}$ ) were collected from the non-necrotic areas of the tumor foci. TMA sections (4 mm) were constructed using the intratumoral samples from the $296 \mathrm{HCC}$ patients.

\section{In situ hybridization}

In situ hybridization (ISH) was performed using miR-34a 5'-DIG-labeled LNA ${ }^{\mathrm{TM}}$ probes (Exiqon, Vedbaek, Denmark) according to the manufacturer's protocol. The sequence of the detection probe for human mature miR34a was 5' ACAACCAGCTAAGACACTGCCA 3'. TMAs composed of intratumoral tissues from 296 patients were prepared as follows: the TMAs were (1) acetylated with $0.25 \%$ acetic anhydride after the sections were dewaxed and rehydrated; (2) prehybridized in Exiqon hybridization buffer (Exiqon, Vedbæk, Denmark) at $55^{\circ} \mathrm{C}$ for $60 \mathrm{~min}$; (3) incubated with $40 \mu \mathrm{l}$ of a 1:600 dilution of the corresponding 5'-DIG-labeled LNA ${ }^{\mathrm{TM}}$ miRNA probe; (4) washed twice with SSC buffer at $60^{\circ} \mathrm{C}$ for a minimum of $20 \mathrm{~min}$; (5) treated with digoxigenin (DIG) blocking reagent (Invitrogen); (6) incubated with anti-DIG alkaline phosphatase conjugated antibody (Invitrogen) at $4^{\circ} \mathrm{C}$ for $24 \mathrm{~h}$; and (7) washed twice and counterstained slightly with Mayer's hematoxylin.

\section{Evaluation of ISH findings}

The slides were examined by three independent, blinded observers using an Olympus BX51 microscope (Olympus BX51, CCD: DP71, Japan). The intensity of the staining and the percentage of positively stained tumor cells were scored by each observer, and the three scores were averaged [33]. The scoring scale for the percentage of positively stained cells was as follows: none $=0 ;<1 \%=$
$1 ; 1-10 \%=2 ; 10-33 \%=3 ; 34-67 \%=4 ;$ and $>67 \%=5$. The intensity of the staining was scored semiquantitatively as follows: none $=0$; weak $=1$; intermediate $=2$; and strong $=3$. The staining intensity and the percent of positively stained cells scores were summed to yield a final score that ranged from $0-8$. A final score of $0-2$ was designated as negative for miR-34a expression and a final score of 3-8 was designated as positive for miR-34a expression.

\section{Cell culture}

The SMMC-7721, HCCLM3, and MHCC97H human hepatoma cell lines used in this study were purchased from KeyGen (Nanjing KeyGen Biotech Co.Ltd, China), and the HepG2 human hepatoma cells were purchased from the Chinese Academy of Sciences cell bank. The HCCLM3 cells were previously described [34]. All of the cell lines were maintained in DMEM medium (GIBCO, Carlsbad, CA, USA) supplemented with $10 \%$ fetal bovine serum, $80 \mathrm{U} / \mathrm{ml}$ of penicillin sodium, and $0.08 \mathrm{mg} / \mathrm{ml}$ of streptomycin sulfate at $37^{\circ} \mathrm{C}$ in humidified air containing $5 \%$ carbon dioxide.

\section{Oligonucleotide transfection}

The miRIDIAN miR-34a Hairpin Inhibitor, Mimic, and negative control oligonucleotides (Dharmacon, GE Healthcare) were used to inhibit and restore miR-34a function. These oligonucleotides were transfected into HCC cells using Lipofectamine ${ }^{\mathrm{TM}} 2000$ according to the manufacturer's instructions (Invitrogen).

\section{In vitro cell proliferation assays}

Cell proliferation was detected using the Cell Counting Kit-8 (KeyGen Biotech Co.Ltd, China) assay. In brief, SMMC-7721 and HCCLM3 cells were seeded into 96-well plates at an initial density of $1 \times 10^{4}$ cells per well. The oligonucleotides were transfected into the HCC cells $24 \mathrm{~h}$ later, and at 0, 24, 48, 72, and $96 \mathrm{~h}$ after oligonucleotide transfection, $10 \mu \mathrm{l}$ of the kit reagent was added to each well. All of the plates were scanned at 450 $\mathrm{nm} 2 \mathrm{~h}$ later using a microplate reader (Molecular Devices, USA) and the cell proliferation value was calculated based on the resulting OD value.

\section{In vitro migration and invasion assays}

Cell migration and invasion were analyzed using 24-well transwell plates (8- $\mu \mathrm{m}$ pore size, Corning, USA). For the transwell migration assays, $5 \times 10^{4}$ cells were seeded in the upper chamber of the plate with a non-coated membrane. For the invasion assays, $1.0 \times 10^{5}$ cells were seeded in the upper chamber of the plate with a Matrigel ${ }^{\circledR}$ coated membrane (Corning, USA). The cells were seeded in $1 \%$ serum medium, and $10 \%$ serum medium was used 
as the chemoattractant in the lower chamber of the plate. After incubation at $37^{\circ} \mathrm{C}$ for 24 or $48 \mathrm{~h}$, nonmigratory and noninvasive cells in the upper chambers were removed using cotton wool. The invading cells on the undersides of the membranes were fixed in $100 \%$ methanol for 10 min, stained with Giemsa (Jiancheng, Nanjing, China), and counted by microscopy. Sixty hours prior to plating, miR-34a inhibitors, mimics, or negative controls were transfected into cells at a final concentration of $100 \mathrm{nM}$ using Lipofectamine ${ }^{\mathrm{TM}} 2000$.

\section{Statistical analysis}

All of the statistical analyses were performed using IBM SPSS Statistics 20. The Fisher's exact test was used to compare qualitative variables, and quantitative variables were analyzed using Pearson's correlation test. To determine whether there was an association between the expression of the candidate molecule and a higher incidence of BM, we analyzed censored time-to-event data using a log-rank test and a Cox regression model. A twotailed $P<0.05$ was considered significant. A correction for multiple hypotheses testing was performed using the false discovery rate (FDR).

\section{ACKNOWLEDGMENTS}

The authors thank Shanghai Biochip Company Ltd. for expert technical assistance.

\section{CONFLICTS OF INTEREST}

No potential conflicts of interest were disclosed.

\section{FUNDING}

This work was supported by grant no. 30973500 from the National Natural Science Foundation of China and grant no.20124208 from the General Program Foundation of the Shanghai Municipal Bureau of Health.

\section{REFERENCES}

1. Torre LA, Bray F, Siegel RL, Ferlay J, Lortet-Tieulent J, Jemal A. Global cancer statistics, 2012. CA Cancer J Clin. 2015; 65:87-108.

2. El-Serag, H. B. Epidemiology of viral hepatitis and hepatocellular carcinoma. Gastroenterology. 2012; 142:1264-73.

3. Natsuizaka M, Omura T, Akaike T, Kuwata Y, Yamazaki K, Sato T, Karino Y, Toyota J, Suga T, Asaka M. Clinical features of hepatocellular carcinoma with extrahepatic metastases. J Gastroenterol Hepatol. 2005; 20: 1781-7.

4. Xiang ZL, Zeng ZC, Tang ZY, Fan J, He J, Zeng HY, Zhu $\mathrm{XD}$. Potential prognostic biomarkers for bone metastasis from hepatocellular carcinoma. Oncologist. 2011;16: 1028-39.

5. Roodman GD. Mechanisms of bone metastasis. N Engl J Med. 2004;350: 1655-64.

6. Aapro M, Abrahamsson PA, Body JJ, Coleman RE, Colomer R, Costa L, Crinò L, Dirix L, Gnant M, Gralow J, Hadji P, Hortobagyi GN, Jonat W, et al. Guidance on the use of bisphosphonates in solid tumours: recommendations of an international expert panel. Ann Oncol. 2008; 19:420-32.

7. Xiang ZL, Zeng ZC, Tang ZY, Fan J, Zhuang PY, Liang Y, Tan YS, He J. Chemokine receptor CXCR4 expression in hepatocellular carcinoma patients increases the risk of bone metastases and poor survival. BMC Cancer. 2009;9:176.

8. Xiang ZL, Zeng ZC, Fan J, Tang ZY, Zeng HY. Expression of connective tissue growth factor and interleukin-11 in intratumoral tissue is associated with poor survival after curative resection of hepatocellular carcinoma. Mol Biol Rep. 2012; 39:6001-6.

9. Xiang ZL, Zeng ZC, Fan J, Wu WZ, He J, Zeng HY, Tang ZY. A clinicopathological model to predict bone metastasis in hepatocellular carcinoma. J Cancer Res Clin Oncol. 2011; 137:1791-7.

10. He J, Zeng ZC, Tang ZY, Fan J, Zhou J, Zeng MS, Wang JH, Sun J, Chen B, Yang P, Pan BS. Clinical features and prognostic factors in patients with bone metastases from hepatocellular carcinoma receiving external beam radiotherapy. Cancer. 2009;115:2710-20.

11. Marino MT, Grilli A, Baricordi C, Manara MC, Ventura S, Pinca RS, Bellenghi M, Calvaruso M, Mattia G, Donati D, Tripodo C, Picci P, Ferrari S, et al. Prognostic significance of miR-34a in Ewing sarcoma is associated with cyclin D1 and ki-67 expression. Ann Oncol. 2014; 25:2080-6.

12. Yu G, Yao W, Xiao W, Li H, Xu H, Lang B. MicroRNA-34a functions as an anti-metastatic microRNA and suppresses angiogenesis in bladder cancer by directly targeting CD44. J Exp Clin Cancer Res. 2014; 33:779.

13. Nie J, Ge X, Geng Y, Cao H, Zhu W, Jiao Y, Wu J, Zhou J, Cao J. miR-34a inhibits the migration and invasion of esophageal squamous cell carcinoma by targeting Yin Yang1. Oncol Rep. 2015;34:311-7.

14. Peurala H, Greco D, Heikkinen T, Kaur S, Bartkova J, Jamshidi M, Aittomäki K, Heikkilä P, Bartek J, Blomqvist C, Bützow R, Nevanlinna H. MiR-34a expression has an effect for lower risk of metastasis and associates with expression patterns predicting clinical outcome in breast cancer. PLoS One. 2011;6:e26122.

15. Wu J, Wu G, Lv L, Ren YF, Zhang XJ, Xue YF, Li G, Lu $X$, Sun Z, Tang KF. MicroRNA-34a inhibits migration and invasion of colon cancer cells via targeting to Fra-1. Carcinogenesis. 2012; 33:519-28.

16. Jamieson NB, Morran DC, Morton JP, Ali A, Dickson EJ, Carter CR, Sansom OJ, Evans TR, McKay CJ, Oien KA. MicroRNA molecular profiles associated with diagnosis, 
clinicopathologic criteria, and overall survival in patients with resectable pancreatic ductal adenocarcinoma. Clin Cancer Res. 2012; 18:534-45.

17. Zhang H, Li S, Yang J, Liu S, Gong X, Yu X. The prognostic value of miR-34a expression in completely resected gastric cancer: tumor recurrence and overall survival. Int J Clin Exp Med. 2015; 8:2635-41.

18. Gao H, Zhao H, Xiang W. Expression level of human miR-34a correlates with glioma grade and prognosis. J Neurooncol. 2013; 113:221-8.

19. Genovese G, Ergun A, Shukla SA, Campos B, Hanna J, Ghosh P, Quayle SN, Rai K, Colla S, Ying H, Wu CJ, Sarkar $\mathrm{S}$, Xiao $\mathrm{Y}$, et al. MicroRNA regulatory network inference identifies miR-34a as a novel regulator of TGF- $\beta$ signaling in glioblastoma. Cancer Discov. 2012 ; 2:736-49.

20. Fang C, Zhu DX, Dong HJ, Zhou ZJ, Wang YH, Liu L, Fan L, Miao KR, Liu P, Xu W, Li JY. Serum microRNAs are promising novel biomarkers for diffuse large B cell lymphoma. Ann Hematol. 2012; 91: 553-9.

21. Schultz NA, Dehlendorff C, Jensen BV, Bjerregaard JK, Nielsen KR, Bojesen SE, Calatayud D, Nielsen SE, Yilmaz M, Holländer NH, Andersen KK, Johansen JS. MicroRNA biomarkers in whole blood for detection of pancreatic cancer. JAMA. 2014; 311:392-404.

22. Kubiczkova L, Kryukov F, Slaby O, Dementyeva E, Jarkovsky J, Nekvindova J, Radova L, Greslikova H, Kuglik P, Vetesnikova E, Pour L, Adam Z, Sevcikova S, et al. Circulating serum microRNAs as novel diagnostic and prognostic biomarkers for multiple myeloma and monoclonal gammopathy of undetermined significance. Haematologica. 2014; 99:511-8.

23. Patel D, Boufraqech M, Jain M, Zhang L, He M, Gesuwan K, Gulati N, Nilubol N, Fojo T, Kebebew E. MiR-34a and miR-483-5p are candidate serum biomarkers for adrenocortical tumors. Surgery. 2013; 154:1224-8.

24. Krzeszinski JY, Wei W, Huynh H, Jin Z, Wang X, Chang TC, Xie XJ, He L, Mangala LS, Lopez-Berestein G, Sood AK, Mendell JT, Wan Y. miR-34a blocks osteoporosis and bone metastasis by inhibiting osteoclastogenesis and Tgif2. Nature. 2014; 512:431-5.

25. Chen WY, Liu SY, Chang YS, Yin JJ, Yeh HL, Mouhieddine TH, Hadadeh O, Abou-Kheir W, Liu YN. MicroRNA34a regulates WNT/TCF7 signaling and inhibits bone metastasis in Ras-activated prostate cancer. Oncotarget. 2015; 6:441-57. doi: 10.18632/oncotarget.2690.
26. Gougelet A, Sartor C, Bachelot L, Godard C, Marchiol C, Renault G, Tores F, Nitschke P, Cavard C, Terris B, Perret C, Colnot S. Antitumour activity of an inhibitor of miR-34a in liver cancer with $\beta$-catenin-mutations. Gut. 2016;65:1024-34.

27. Yang P, Li QJ, Feng Y, Zhang Y, Markowitz GJ, Ning S, Deng Y, Zhao J, Jiang S, Yuan Y, Wang HY, Cheng SQ, Xie D, et al. TGF- $\beta$-miR-34a-CCL22 signaling-induced Treg cell recruitment promotes venous metastases of HBVpositive hepatocellular carcinoma. Cancer Cell. 2012; 22:291-303.

28. Kroh EM, Parkin RK, Mitchell PS, Tewari M. Analysis of circulating microRNA biomarkers in plasma and serum using quantitative reverse transcription-PCR (qRT-PCR). Methods. 2010; 50:298-301.

29. Landgraf P, Rusu M, Sheridan R, Sewer A, Iovino N, Aravin A, Pfeffer S, Rice A, Kamphorst AO, Landthaler M, Lin C, Socci ND, Hermida L, et al. A mammalian microRNA expression atlas based on small RNA library sequencing. Cell. 2007; 129:1401-14.

30. Xiang ZL, Zeng ZC, Tang ZY, Fan J, Sun HC, Wu WZ, Tan YS. Increased expression of vascular endothelial growth factor-C and nuclear CXCR4 in hepatocellular carcinoma is correlated with lymph node metastasis and poor outcome. Cancer J. 2009; 15:519-25.

31. Xiang ZL, Zeng ZC, Fan J, Tang ZY, Zeng HY, Gao DM. Gene expression profiling of fixed tissues identified hypoxia-inducible factor- $1 \alpha$, VEGF, and matrix metalloproteinase-2 as biomarkers of lymph node metastasis in hepatocellular carcinoma. Clin Cancer Res. 2011; 17:5463-72.

32. Xiang ZL, Zeng ZC, Fan J, Tang ZY, He J, Zeng HY, Chang JY. The expression of HIF-1 $\alpha$ in primary hepatocellular carcinoma and its correlation with radiotherapy response and clinical outcome. Mol Biol Rep. 2012; 39:2021-9.

33. He M, Gao L, Zhang S, Tao L, Wang J, Yang J, Zhu M. Prognostic significance of miR-34a and its target proteins of FOXP1, p53, and BCL2 in gastric MALT lymphoma and DLBCL. Gastric Cancer. 2014;17:431-41.

34. Li Y, Tian B, Yang J, Zhao L, Wu X, Ye SL, Liu YK, Tang ZY. Stepwise metastatic human hepatocellular carcinoma cell model system with multiple metastatic potentials established through consecutive in vivo selection and studies on metastatic characteristics. J Cancer Res Clin Oncol. 2004; 130:460-8. 